\title{
Impact of Nasal Trauma on Olfactory Function
}

\author{
Süleyman Emre Karakurt, Zekiye Orhan, Mehmet Fatih Karakus, Mehmet Ali Cetin, Aykut Ikinciogullari and \\ Huseyin Dere
}

Department of Ear, Nose, Throat, Ankara Numune Training and Research Hospital, Turkey

\begin{abstract}
Objective: To determine the impact of nasal trauma with and without the potential to produce nasal fracture on the olfactory function.

Study Design: A descriptive analytical study.

Place and Duration of Study: Ear, Nose, Throat Clinic, Ankara Numune Training and Research Hospital, Ankara from October 2018 to June 2019.

Methodology: The study included patients with nasal trauma and control subjects. The patients with nasal trauma were divided into two groups as fracture group (Group $F, n=83$ ) and non-fracture group (Group Non- $\mathrm{F}, \mathrm{n}=30$ ). The Group $\mathrm{F}$ was further divided into two subgroups according the presence as septal fracture as Group SF (patients with septal fracture) and Group Non-SF (patients with non-septal fracture). The smell functions of all participants were evaluated using the Sniffin' Sticks test. The odour scores of Group F and Group Non-F were compared versus control group, using the independent sample t-test or Mann-Whitney U-test. Percentage of patients with olfactory dysfunction was compared between Group F and Group Non-F and between Group SF and Group Non-SF using the Chi-square test.

Results: There were a total of 113 participants with mean age of $35.64 \pm 10.44$ years. The median TDI score of Group F was significantly lower in comparison to control group, no significant difference was found between Group Non- $F$ and control group in terms of median TDI score. There was a significant difference between Group F and Group Non-F in terms of the percentage of patients with olfactory dysfunction. No significant difference was found between Group F and Group Non-F with respect to the percentage of patients with olfactory dysfunction.
\end{abstract}

Conclusion: Nasal trauma can lead to olfactory dysfunction only if it has the potential to produce a nasal fracture.

Key Words: Smell disorders, Nasal bone, Bone fractures, Trauma.

How to cite this article: Karakurt SE, Orhan Z, Karakus MF, Cetin MA, Ikinciogullari A, Dere H. Impact of Nasal Trauma on Olfactory Function. J Coll Physicians Surg Pak 2020; 30(09):912-916.

\section{INTRODUCTION}

In the most prominent facial feature, the nose is particularly vulnerable to external injuries. Nasal trauma may cause nasal fracture as well as transient minimal changes in the soft tissues without a fracture. Nasal fractures are the most common type of facial bone fractures. ${ }^{1}$ Nasal fractures may occur alone or in combination with otherfacial bone fractures.

The sense of smell has an important role in daily life; and impairment of this sense may potentially affect an individual's quality of life. ${ }^{2}$ Since the anatomic structures are responsible for smell perception, transmission and processing are located in the upper and middle third of the face; any fractures involving these sites may lead to olfactory disturbances. ${ }^{3}$

Correspondence to: Dr. Süleyman Emre Karakurt, Department of Ear, Nose, Throat, Ankara Numune Training and Research Hospital, Turkey

E-mail: suleymanemrekarakurt@gmail.com

Received: July 03, 2020; Revised: July 29, 2020;

Accepted: September 26, 2020

DOI: https://doi.org/10.29271/jcpsp.2020.09.912
Posttraumatic olfactory dysfunction may result from sinonasal disruption, stretching or shearing of olfactory nerve fibers and contusion or hemorrhage within the olfactory bulb or cortex. ${ }^{4-10}$

Olfactory dysfunction related to head trauma has been extensively studied, with marked variations in prevalence estimates reported for olfactory dysfunction after head trauma. ${ }^{11-13}$ Olfactory dysfunction due to trauma may occur due to central or peripheral mechanisms. Olfactory nerve damage at the level of the cribriform plate is considered among the peripheral mechanisms. Olfactory nerve damage can also occur due to the stretching of the nerve at this level with the effect of trauma, without a cribriform plate fracture. For that reason, nerve damage and olfactory dysfunction can occur without fracture of the cribriform plate. There are relatively a low number of studies in literature that investigated the impact of facial bone fractures including nasal fractures on the sense of smell and the effect of nasal injuries not associated with nasal fractures on the olfactory function; it has not beenassessed in almostall of the publications. ${ }^{1,3,14,15}$

The aim of the current study was to examine the impact of nasal trauma with and without the potential to produce nasal fracture on theolfactory function. 
Table I: Comparison of the odour scores among the groups.

\begin{tabular}{|l|c|c|c|c|c|}
\hline & $\begin{array}{c}\text { Group F } \\
\text { Mean } \pm \text { S.D/Median (IQR) }\end{array}$ & $\begin{array}{c}\text { Group non-F } \\
\text { Mean } \pm \text { S.D/Median (IQR) }\end{array}$ & $\begin{array}{c}\text { Control group } \\
\text { Mean } \pm \text { S.D/Median (IQR) }\end{array}$ & $\begin{array}{c}\text { p-value } \\
\text { (Group F vs. Control Group) }\end{array}$ & $\begin{array}{c}\text { p-value } \\
\text { (Group Non-F vs. Control } \\
\text { Group) }\end{array}$ \\
\hline OT & $5.60 \pm 2.41 / 5.38(3.81$ & $8.78 \pm 2.80 / 8.25(4.25)$ & $8.96 \pm 2.63 / 8.63(3)$ & $<0.001^{u}$ & $0.798^{\mathrm{t}}$ \\
\hline OD & $11.94 \pm 3.01 / 12(4.25)$ & $13.24 \pm 2.28 / 14(3)$ & $13.67 \pm 2.32 / 14(2.25)$ & $0.010^{\mathrm{u}}$ & $0.350^{\mathrm{u}}$ \\
\hline OI & $10.35 \pm 2.40 / 11(3)$ & $11.34 \pm 2.19 / 11(3)$ & $12.37 \pm 2.30 / 12(3.25)$ & $0.001^{\mathrm{u}}$ & $0.086^{\mathrm{t}}$ \\
\hline TDI & $27.94 \pm 4.66 / 28.13(7.31)$ & $33.36 \pm 3.41 / 33(4)$ & $34.78 \pm 3.27 / 34.63(6.38)$ & $<0.001^{u}$ & $0.123^{\mathrm{u}}$ \\
\hline \multicolumn{7}{|l|}{$t:$ Independent sample t-test and Mann-Whitney U-test; OT: Odour threshold; OD: Odour discrimination; Ol: Odour identification; TDI: Threshold-discrimination-identification. } \\
\hline
\end{tabular}

Table II: Distribution of patients with olfactory dysfunction among the groups.

\begin{tabular}{|c|c|c|c|c|}
\hline & & Normosmia & Hyposmia & p-value \\
\hline \multirow{2}{*}{$\begin{array}{l}\text { Patient group } \\
n(\%)\end{array}$} & Group F & $26(48.1 \%)$ & $28(51.9 \%)$ & \multirow{2}{*}{0.006} \\
\hline & Group Non-F & $23(79.3 \%)$ & $6(20.7 \%)$ & \\
\hline \multirow{2}{*}{$\begin{array}{l}\text { Group F } \\
\mathrm{n}(\%)\end{array}$} & Group SF & $10(40 \%)$ & $15(60 \%)$ & \multirow{2}{*}{0.266} \\
\hline & Group Non-SF & 16 (55.2\%) & $13(44.8 \%)$ & \\
\hline
\end{tabular}

\section{METHODOLOGY}

Ethics approval was obtained from the Institutional Review Board (No. E-18-2225). All participants signed informed consent. The study was conducted with a total of 113 subjects, including 83 adult patients presenting to Ear, Nose, Throat Clinic, Ankara Numune Training and Research Hospital, between October 2018 and June 2019 with a history of nasal trauma with or without fracture and 30 gender and age matched control subjects with no known smell disturbance and history of nasal trauma. Subjects of either gender, over 18 years of age, were included in the study. Subjects with a history of an upper respiratory tract infection within the previous month, patients with known olfactory dysfunction, patients with a history of neurological or psychiatric illness, patients with a metabolic, endocrine condition or chronic sinonasal disease and tobacco users were excluded from the study. Additionally, patients with nasal fractures related to car accidents or falls and patients with nasal fractures together with other cranial and/or facial fractures were also excluded. All participants underwent otolaryngological examination and olfactory function tests and also computed tomography (CT) imaging for those with a history of trauma.

The patients with a history of nasal trauma were divided into two groups as Group F (patient group in which nasal trauma causes nasal fracture with or without septal fracture) and Group Non-F (patient group in which nasal trauma does not cause nasal or septal fracture) based on the presence of a nasal fracture as identified by physical examination and CT findings. Patients with no known olfactory disturbances were included as control group. The Group F was further divided in two subgroups, according to the presence as septal fracture as Group SF (group of patients with nasal fracture with septal fracture) and Group Non-SF (group of patients with nasal fracture without septal fracture). The Group F, Group Non- $F$, and control group were compared in terms of percentage distribution of mean age and gender. The mean odour scores of Group F and Group Non-F were compared with the mean odour scores of control group. Group F and Group Non-F were compared with respect to the percentage of patients with olfactory dysfunction. Similarly, the percentage of patients with olfactory dysfunction was also compared between Group SF and Group Non-SF.

Olfactory function tests were conducted within 10-day period from the time of nasal trauma to nasal fracture reduction. Olfactory function was assessed using the Sniffin' Sticks test (Burghart $\mathrm{GmbH}$, Wedel, Germany). All smell tests were performed by the same investigator. For odour presentation, after removing the cap of a felt pen impregnated with an odourant, the tip of the pen was positioned about $2 \mathrm{~cm}$ from the subject's nostrils and the subject was asked to sniff.

The Sniffin' Sticks test consists of an odour threshold (T) test, an odour discrimination (D) test and an odour identification (I) test. With the odour threshold test, the concentration at which the patient defines the threshold for $n$-butanol is determined. The odour discrimination test is conducted with two pens impregnated with the same odourant and one pen with a different odour; and the subject is asked to identify the pen with a different odour. The odour identification test is performed using 16 well-known odours and the subject is presented 4 choices and required to identify the odour that he/she sniffed. The scores from the odour threshold test $(O T)$, the odour discrimination test (OD) and the odour identification test (OI) are obtained individually. These scores are summed up to obtain the total threshold-discrimination-identification (TDI) score. TDI score of 30 or less was defined as hyposmia and a score greater than 30 was defined as normosmia, in accordance with the recommended test procedure.

The Shapiro-Wilk test was used to assess data distribution. Continuous variables were presented as mean with standard deviation or median with interquartile range. Categorical variables were presented as number with percentage. Cate- 
gorical variables were analysed using the Chi-square test. The difference between the means of two independent groups was assessed using the independent sample t-test or Mann-Whitney U-test. The Kruskal-Wallis test was used to compare means among groups. A p-value less than 0.05 was considered statistically significant. The SPSS statistical software (SPSS for Windows version 21.0; SPSS Inc., Chicago, IL) was used for statistical analyses.

\section{RESULTS}

The study sample consisted of 44 (38.9\%) females and 69 (61.1\%) males. The mean age of all subjects was $35.64 \pm 10.44$ years. Group F, Group Non-F, and control group comprised 54 (47.8\%), 29 (25.7\%) and 30 (26.5\%) subjects, respectively. There were 25 (46.3\%) patients in Group SF and 29 (53.7\%) patients in Group Non-SF.

The median ages of Group F, Group Non-F, and control group were 34.50 (21.25), 37 (16) and 32 (9.75) years, respectively; with no significant difference in the median age among the groups $(p=0.585)$. Group $F$ consisted of 17 (31.5 $\%$ ) females and 37 (68.5\%) males. Group Non-F consisted of $13(44.8 \%)$ females and $16(55.2 \%)$ males, and control group comprised 14 (46.7\%) females and 16 (53.3\%) males. The percentage distribution of gender was not significantly different among the groups $(p=0.295)$.

The median OT scores were 5.38 (3.81), 8.25 (4.25) and 8.63 (3) for Group F, Group Non-F and control group (Table I). While Group $\mathrm{F}$ had a median OT score that was significantly lower than control group. No significant difference was found between Group Non-F and control group in the mean OT score ( $p$ values $<0.001$ and 0.798 , respectively, Table I). Group F, Group Non-F and control group had median OD scores of 12 (4.25), 14 (3) and 14 (2.25), respectively, Table I. The median OD score of Group F was significantly lower versus control group, but not significantly different between Group Non-F and control group ( $p$ values 0.01 and 0.350, respectively, Table I). Group F, Group Non-F and control groups had median Ol scores of 11 (3), 11 (3) and 12 (3.25), respectively, Table I. The median Ol score of Group $\mathrm{F}$ was significantly lower compared to control group $(p=0.001$, Table I). The median TDI scores of Group F, Group Non-F and control group were 28.13 (7.31), 33 (4) and 34.63 (6.38), respectively, Table I. While the median TDI score of Group $F$ was significantly lower in comparison to control group, no significant difference was found between Group Non-F and control group in terms of median TDI score ( $p$ values $<0.001$ and 0.123 , respectively, Table I).

There were 26 (48.1\%) normosmic and 28 (51.9\%) hyposmic patients in Group F and 23 (79.3\%) normosmic and 6 $(20.7 \%)$ hyposmic patients in Group Non-F, Table II. Group F had a statistically significantly higher percentage of hyposmic patients in comparison to Group Non-F ( $p=0.006$, Table II). In the Group SF, 10 (40\%) patients were normosmic and 15 (60\%) patients were hyposmic and the corresponding figures were 16 (55.2\%) and 13 (44.8\%), respectively in Group Non-SF (Table II). No significant difference was observed between Group SF and Group Non-SF in terms of the percentage of normosmic and hyposmic patients $(p=0.266$, Table II).

\section{DISCUSSION}

The present findings showed that olfactory dysfunction (OD) occurred after nasal trauma associated with a nasal fracture, but not after nasal trauma not associated with a nasal fracture. The coexistence of septal fracture with a nasal fracture did not have an impact on the occurrence of olfactory dysfunction.

Ageing affects all aspects of detecting odours. It was reported that odour discrimination and odour identification deteriorate after the age of 20 and there is a rapid decline in the odour threshold by 50 years and onwards. ${ }^{16}$ Gender is another factor that affects the sense of smell. In our study, no significant difference was found among the groups in terms of median age and gender distribution.

Published studies that investigated the impact of head trauma on the olfactory function indicate that the sense of smell is adversely affected by trauma. ${ }^{11-13}$ In one study exploring the frequency of olfactory dysfunction after traumatic brain injury, olfactory dysfunction was reported in $13.7 \%$ and anosmia in $8.2 \%$ of the participants. ${ }^{11}$ In a separate study examining the effect of mild traumatic brain injury on the sense of smell, olfactory dysfunction was identified in $33.5 \%$ of the patients and of these patients with OD $87 \%$ had anosmia and $13 \%$ had hyposmia. ${ }^{12}$ Although the reported rates have been variable, there is a general consensus that head trauma causes olfactory impairment. The mechanisms underlying olfactory dysfunction after traumatic head injury are complex and involves both central and peripheral mechanisms. ${ }^{11}$ The mechanism, through which central lesions occur, has a wide spectrum ranging from intracranial hemorrhage and axonal damage and may involve olfactory centers and olfactory pathways in the brain. ${ }^{17-19}$ On the other hand, peripheral nerve injury is caused by peripheral mechanisms. ${ }^{20-23}$

The effect of nasal trauma on olfactory function is a relatively less studied topic, with a limited number of studies available in the literature. Almost all of these studies did not investigate olfactory function after nasal injuries not potentially associated with a nasal fracture. In a study by Kim et al. which evaluated the impact of nasal bone fractures on olfactory dysfunction, ${ }^{1}$ nearly one-half of the patients exhibited post-traumatic olfactory dysfunction and had no improvement in olfaction at 6 months after closed reduction. They concluded that the resulting olfactory dysfunction may be due to factors other than the fracture itself; and suggested that the association between injuries that cause 
nasal fracture and the mechanism behind posttraumatic olfactory dysfunction should be further investigated. ${ }^{1}$ In another study, the medical records of 143 patients with nasal trauma were reviewed retrospectively; and the overall prevalence of olfactory dysfunction was reported at $6.3 \%$ and of the patients with olfactory dysfunction, about $80 \%$ had recovery of olfactory function. ${ }^{14}$ van Damme et al. investigated the impact of high central midface fractures on the sense of smell and reported that more smell disturbances are found with the increased severity of the trauma. ${ }^{15}$ In that study, smell disturbances were identified in $25 \%$ of the nasal fractures and $80 \%$ of the fronto-nasal-Le Fort fractures..$^{15}$ In contrast to the aforementioned studies, Renzi et $a l .^{3}$ questioned a sample of 92 patients with upperthird or middle-third facial fractures including 4 patients with pure nasal fracture to estimate smell disturbance and reported no smell disturbance in the latter patients. In our study, patients with a pure nasal fracture (Group F) had significantly lower TDI score in comparison to control group and $51.9 \%$ of these patients were found to have olfactory dysfunction.

The studies mentioned above clearly indicate that the mechanism by which olfactory dysfunction occurs after a nasal fracture merits further investigation. As opposed to smell disturbance following head trauma, olfactory dysfunction after nasal injuries may occur as a result of the impact of the trauma as well as intranasal anatomical changes secondary to a nasal fracture. A change in nasoseptal structures may block transmission of odour molecules to the olfactory area, leading to olfactory dysfunction. Complete or partial loss of olfactory function may occur as a result of obstruction of nasal passages or damage to the olfactory nerve. ${ }^{24}$ In other words, olfactory dysfunction may involve conductive defects or sensorineural defects. However, it has not yet been clarified which of these mechanisms is more effective. Considering this gap of knowledge, we aimed to evaluate olfactory function in patients with nasal trauma not potentially associated with nasal fracture and found no significant difference in TDI scores between the patient group (Group Non-F) and control group. The percentage of patients with olfactory dysfunction was significantly lower in this group than in the group of patients with nasal trauma, potentially associated with nasal fracture (Group F). This finding was interpreted as evidence that nasal trauma can lead to olfactory dysfunction only if it has the potential to produce a nasal fracture. Moreover, no difference was found in the percentage distribution of olfactory dysfunction between patients with septal fracture (Group SF) and patients without septal fracture (Group Non-SF) in the patient group with nasal fracture (Group F).This suggests that nasal trauma, potentially leading to a nasal fracture, causes olfactory dysfunction as a result of the damage to sensorineural system associated with the trauma rather than anatomical changes in the nasal structures secondary to the impact of the trauma.
Olfactory function is a topic that should be explored in all aspects including the threshold, discrimination and identification functions. In contrast to the odour threshold, odour discrimination and identification areas are suprathreshold tests. While the odour threshold represents peripheral olfactory function, discrimination and identification domains reflect higher central nervous system functions responsible for odour processing..$^{25}$ In the present study, patients with nasal fracture showed significantly lower OT, OD and OI scores compared to control group. This suggests that olfactory dysfunction caused by a nasal fracture involves both peripheral and central olfactory pathways.

With the current study, the impact of nasal trauma, potentially associated or not associated with nasal fractures on olfactory function, was evaluated in detail for the first time, including threshold, discrimination and identification areas. A major limitation of this study was that olfactory function following nasal fracture reduction was not assessed.

\section{CONCLUSION}

Nasal trauma can lead to olfactory dysfunction only if it has the potential to produce a nasal fracture. Therefore, nasal trauma potentially leading to a nasal fracture seems to cause olfactory dysfunction as a result of the damage to sensorineural system associated with the trauma rather than anatomical changes in the nasal structures secondary to the impact of the trauma.

\section{ETHICAL APPROVAL:}

Ethics approval was obtained from the Institutional Review Board (No. E-18-2225).

\section{PATIENTS' CONSENT:}

All participants signed informed consent.

\section{CONFLICT OF INTEREST:}

The authors report no conflict of interest.

\section{AUTHORS' CONTRIBUTION:}

SEK: Study conception and design, acquisition of data. ZO: Study conception and design, acquisition of data.

MFK: Analysis and interpretation of data, drafting of manuscript. MAC: Study conception and design, analysis and interpretation of data.

Al: Study conception and design, analysis and interpretation of data.

HHD: Acquisition of data, drafting of manuscript.

\section{REFERENCES}

1. Kim SW, Park B, Lee TG, Kim JY. Olfactory dysfunction in nasal bone fracture. Arch Craniofac Surg 2017; 18(2):92-6. doi: 10.7181/acfs.2017.18.2.92.

2. Khilji MS, Tahir M, Jafari FH. Morphological and quantitative features of mitral cells in the rat olfactory bulb after administration of propranolol. J Coll Physicians Surg Pak 2010; 
20(4):237-41.

3. Renzi G, Carboni A, Gasparini G, Perugini M, Becelli R. Taste and olfactory disturbances after upper and middle third facial fractures: A preliminary study. Ann Plast Surg 2002; 48(4):355-8. doi: 10.1097/00000637-200204000-00003.

4. Nordin S, Brämerson A. Complaints of olfactory disorders: Epidemiology, assessment and clinical implications. Curr Opin Allergy Clin Immunol 2008; 8(1):10-15. doi: 10.1097/ ACl.0b013e3282f3f473.

5. Doty RL. Olfaction. Annu Rev Psychol 2001; 52:423-52.

6. Fujii M, Fukazawa K, Takayasu S, Sakagami M. Olfactory dysfunction in patients with head trauma. AurisNasus Larynx 2002; 29(1):35-40. doi: 10.1016/s0385-8146(01) 00118-3.

7. Coelho DH, Costanzo RM. Posttraumatic olfactory dysfunction. Auris Nasus Larynx 2016; 43(2):137-43. doi: 10.1016/j.anl.2015.08.006.

8. De Kruijk JR, Leffers P, Menheere PP, Meerhoff S, Rutten J, Twijnstra A. Prediction of post-traumatic complaints after mild traumatic brain injury: early symptoms and biochemical markers. J Neurol Neurosurg Psychiatry 2002; 73(6): 727-32. doi: 10.1136/jnnp.73.6.727.

9. Zusho H. Posttraumatic anosmia. Arch Otolaryngol 1982; 108(2):90-2. doi: 10.1001/archotol.1982.00790500026006.

10. Fan LY, Kuo CL, Lirng JF, Shu CH. Investigation of prognostic factors for post-traumatic olfactory dysfunction. J Chin Med Assoc 2015; 78(5):299-303. doi: 10.1016/j.jcma.2014. 11.009.

11. Bratt $M$, Skandsen $T$, Hummel $T$, Moen KG, Vik A, Nordgård $S$, et al. Frequency and prognostic factors of olfactory dysfunction after traumatic brain injury. Brain Inj 2018; 32(8):1021-7. doi: 10.1080/02699052.2018.

12. Ciofalo A, De Vincentiis M, Iannella G, Zambetti G, Giacomello P, Altissimi G, et al. Mild traumatic brain injury:evaluation of olfactory dysfunction and clinical-neurological characteristics. Brain Inj 2018; 32(5):550-6. doi: 10.1080/02699052.2018.1432074

13. Frasnelli J, Laguë-Beauvais M, LeBlanc J, Alturki AY, Champoux MC, Couturier $C$, et al. Olfactory function in acute traumatic brain injury. Clin Neurol Neurosurg 2016; 140:68-72. doi: 10.1016/j. clineuro.2015.11.013.

14. Dursun E, Battal B. Olfactory dysfunction following nasal trauma. Int J Otorhinolaryngol 2008; 10:1-4.

15. Van Damme PA, Freihofer HP. Disturbances of smell and taste after high central midface fractures. J Craniomaxillofac Surg 1992; 20(6):248-50. doi: 10.1016/s1010-5182 (05)80436-6.

16. Schlosser RJ, Desiato VM, Storck KA, Nguyen SA, Hill JB, Washington $\mathrm{BJ}$, et al. A Community-Based Study on the Prevalence of Olfactory Dysfunction. Am J Rhinol Allergy 2020; 34(5):661-70.1945892420922771.

17. Smith DH, Meaney DF, Shull WH. Diffuse axonal injury in head trauma. J Head Trauma Rehabil 2003; 18(4):307-16.

18. Skandsen T, Kvistad KA, Solheim O, Strand IH, Folvik M, Vik A. Prevalence and impact of diffuse axonal injury in patients with moderate and severe head injury: A cohort study of earlymagnetic resonance imaging findings and 1-year outcome. J Neurosurg 2010; 113(3):556-63. doi: 10.3171/ 2009.9.JNS09626.

19. Povlishock JT, Katz DI. Update of neuropathology and neurological recovery after traumatic brain injury. J Head Trauma Rehabil 2005; 20(1):76-94. doi: 10.1097/ 00001199200501000-00008.

20. Sigurdardottir S, Andelic N, Skandsen T, Anke A, Roe C, Holthe 00, et al. Olfactory Identification and its relationship to executive functions, memory, and disability 1 year after severe traumatic brain injury. Neuropsychology 2015; 30(1):98-108. doi: 10.1037/neu0000206.

21. Lotsch J, Reither N, Bogdanov V, Hahner A, Ultsch A, Hill K, et al. A brain-lesion pattern-based algorithm for the diagnosis of posttraumatic olfactory loss. Rhinology 2015; 53(4):365-70. doi: 10.4193/Rhin15.010.

22. Yousem DM, Geckle RJ, Bilker WB, McKeown DA, Doty RL. Posttraumatic olfactory dysfunction:MR and clinical evaluation. Am J Neuroradiol 1996; 17(6):1171-9.

23. Reiter ER, DiNardo LJ, Costanzo RM. Effects head injury on olfaction andtaste. Otolaryngol Clin North Am 2004; 37(6):1167-84. doi: 10.1016/j.otc.2004.06.005.

24. Costanzo RM, Miwa T. Posttraumatic olfactory loss. Adv Otorhinolaryngol 2006; 63:99- 107. doi: 10.1159/0000 93753.

25. Hummel T, Whitcroft KL, Andrews $P$, Altundag A, Cinghi $C$, Costanzo RM, et al. Position paper on olfactory dysfunction. Rhinol Supp/ 2017; 54(26):1-30. doi: 10.4193/ Rhino16.248. 